\title{
THE ROLE OF TOURIST EMOTION IN DESTINATION LOYALTY
}

\author{
Abdul Hazif Abdul Hamid ${ }^{1}$ \\ Faculty of Entrepreneurship and Business, \\ Universiti Malaysia Kelantan, \\ Malaysia
}

\author{
Mohd Rosli Mohamad \\ Professor \\ Faculty of Entrepreneurship and Business, \\ Universiti Malaysia Kelantan, \\ Malaysia
}

Article DOI: https://doi.org/10.36713/epra3947

\begin{abstract}
Dominant role of tourist emotions as an inescapable element in tourism experience has been widely acknowledged. The purpose of this paper is to develop a conceptual idea on tourist emotion to address inadequacy of the past studies to incorporate characteristic of marketing to determine destination loyalty. Emotion and destination loyalty is an important concept in destination marketing being capable to address switching behavior and variety seeking nature among tourist. This paper discussed various theoretical elements regarding the use of emotion which was originally borrowed from the field of psychology and the need for context specific emotion to accurately capture tourist related emotions. This discussion denotes the key differences in the use of emotion in psychology and tourism. It further proposed a set of direction for future research employing emotion in tourism should be heading in order to better position to predict destination loyalty. This paper also discussed various underpinning theories, limitation and practical implication for academia and policy makers.
\end{abstract}

KEYWORDS: Tourist emotion, loyalty, experience, destination.

\section{INTRODUCTION}

Tourism is one of the fastest growing sectors and seen as major route through which a country can boost its revenues, generate large number of job opportunities to sustain growth rates. Tourism today has been increasingly concerned with the tourist experience of visiting, sightseeing, learning, entertainment and escapism to distress from intense work environment (Awang et al.,2015). Stiff competitions in tourism industry have forced destination managers to adopt relationship marketing as crucial strategy to foster long term engagement in term of loyalty. Tourists emotions were seen as an important element to gain more number of tourist arrival for their respective destination (Rahimi et al.,2017). Increasing efforts have been devoted to examine the ability of emotion to foster better insights on destination loyalty such as tourist retention, reduced marketing cost, repeat visitation, positive word of mouth, and referral (Argan et al.,2014,; Hosany et al.,2015; Prayag et al.,2017).

However, the psychological approach used in past studies to capture tourist emotion in destination marketing has failed to enlighten the richness in destination characteristic and develop intense emotional bonding. This claim can be substantiated by past studies evidencing inconsistent findings to predict destination loyalty when the emotion predictors were not comprehensive predictors and context specific (Burgner et al., 2014; Prayag et al., 2017; Wu et al., 2018). The present study aims to examine the key difference in employing emotion in psychology and tourism by presenting theoretical position expanding the general understanding. This study further proposed emotion in tourism need to be conceptualized beyond psychological process with broader implications on quality of tourist experience aiming for destination loyalty as future direction for tourism research to ensure it can be manipulated as a unique construct incomparable by competitors.

\section{TOURIST EXPERIENCE}

The idea of experience economy derived from theory of experience utility has compelled tourism marketers to exert tourist emotion to be used as hidden persuader that represents a promise that subsequently entice loyalty (Wong et al., 2015). Past research have confirmed that emotionally bonded consumption is less vulnerable to disruption that might induce switching behavior once for all allows strong growth of loyalty (Baksi, 2015; Hosany et al.,2015; Oliver,1999). Furthermore, emotion-based marketing is close to impossible for competitors to imitate because of the unique feel of bonding leading to love for a particular product, brand or services (Gyung Kim \& Mattila, 2013). 
This phenomenon of emotion based loyalty can be explained by theory of experience utility which argues that tourist are more concern to achieve certain gain in utility by patronizing a particular tourism product, brand, place or services even though by nature tourist have variety seeking nature (Barnes et al.,2016). As such, it can be argued that tourists have different attitudes towards loss and gain because the advantages of achieving certain level of utility by repeatedly patronizing a particular destination which stand over psychologically larger. The plausible justification might be, experience in new destination does not necessarily contribute to higher utility as compared to existing destination due to familiarity issues and ability of destination functional setting to fulfil tourist need as well as emotional bonding.

\section{EXPERIENCE AND EMOTION IN DESTINATION}

Tourism offering consist of both primary and secondary features that pull tourist to a particular destination. Tourist encounter with physical environment, service, hospitality as well as other appealing features during visit instigates the experience of emotion or emotional experience as posited by James-Lange Theory ( $\mathrm{Li}$, Scott \& Walters, 2015). There are several theories or general explanation on how emotion works including Lazarus's Cognitive Appraisal Theory, Cannon Bard Theory and Schachter Singer Theory. Emotion theorist views are grounded on the assumption which summarized emotion as combination of physiological arousal, psychological appraisal, cognitive process, subjective experience and expressive behavior (Argan et al.,2014).

All this theoretical position expand the general understanding about emotion which was assumed to be judgment about a situation with clearer denotation that emotion is a unique state of feeling consists of physiological arousal, conscious experience and expressive behavior (Ma et al., 2016). Overall, researcher reached consensus on the notion that emotion is content rich experience that emerge at the level of psychological description which will subsequently lead to appropriate reaction, response or behavior. However, key difference that can be noted at this point when emotion from psychology discipline was applied to tourism marketing is; the field of psychology examines emotion in attempt to explain how emotion works while tourism marketing studied emotion concerning how to use human emotion to effectively market a place, product and services.

Numerous attempts to understand emotional experience have been made over time since the work of Cohen \& Arini (1991) acknowledging the role of affective processes in consumer behavior. In earlier work, most studies focused on tourist' appraisal to place settings such as image and satisfaction but failed to account emotional responses as specific referent that stimulate specific response behaviors (Hosany et al.,2015). Baksi (2015) stated when consuming tourism and leisure services, tourists do not only expect professional services but also desire in satisfying emotional experiences whereby each emotion is a mode or habit of action by itself.

Emotions are an inescapable element in tourism experience and the most relevant component of affect to the travel industry . Wu et al., (2018) defined tourist emotional appraisal as affective responses or immediate emotional responses induced during product/service usage experiences. Emotional appraisal refers to episodes of intense feelings associated with place experience ( Wong et al., 2015) . Tourist emotion plays an important function in defining travel experiences and influencing reactions in term of destination loyalty (Prayag et al., 2017).

The consensus view seems to be that tourist emotion is affective attitudinal reaction stemming from tourist consumption experience during interaction with products, events, culture, specific objects, referents and entire tourism activities. Briefly, it is a concept blending phenomenon regarding consumption, experience and affective response. Researchers have spelled out emotional experience based on its characteristics. For instance, $\mathrm{Li}$ et al.,(2015) viewed emotions arising from consumption experience to be intentional and based on specific objects or referents. Baksi (2015) for example, argued that emotional experience is not merely intentional, but also include tendencies to action. Hosany et al., (2015) described emotion into two categories as normal emotion and consumption emotions with the latter as affective responses to tourist's perceptions of the performance of destination and have been used precisely in tourism research.

Argan et al., (2014) suggested dimensional form of emotional experience from independent response such as joy, love, positive surprise, anger, or fear. Kumar \& Nayak (2015) profiles emotional associations towards destinations to investigate the relationship between emotional profiles, satisfaction, and loyalty. They exhibit five-cluster emotional association towards destination such as impassive, pleased, displeased, mixed, and passionate. Knobloch, Robertson \& Aitken (2016) examine the nature of individual emotional experience and suggested emotion need to be understood beyond hedonic enjoyment with broader implications on well-being and quality of life. Hence, it can be drawn that emotional experience is evaluative reaction mechanism triggered when a person engages in psychological processes.

At first sight, it is important to understand the evaluative reaction of the tourist engaged in tourism and hospitality activities such as vacation, holidays and shopping in order to formulate effective marketing strategies. In common, most researchers 
agree that emotional experience in tourism assembled series of intense feeling, narrower and more specific contextual response on tourist activities. Apparently, the conceptualization of emotional experience in tourism is entirely different from another field. Taking into account various views presented from previous studies, it can be agreed as Wong et al., (2015) stated, that there is slight consistency that can be found among various conceptualization, about the state of a clear definition of emotion is still lacking especially in relation to tourist behavior.

The role of emotion in tourism has received unprecedented recognition in academic fraternity. Many previous research have focus on emotion associated with festivals (Wong et al., 2015; Grappi \& Montanari, 2011), shopping (Donnelly et al., 2013), restaurants (Han \& Jeong, 2013), theme parks (Ali et al., 2016), holidays (Teng \& Liu, 2013; Hosany \& Gilbert, 2010), dark tourism (Kerr \& Price, 2018) and adventure tourism (Faullant, Matzler, \& Mooradian, 2011). Caru \& Cova (2014) explained the time dimension of emotional experience with the use of structural model on how emotions are experienced during pre-consumption, during consumption and post-consumption and how it relates to the behavioral response. Many researchers contended that these outcomes would eventually lead firms to obtain competitive advantages, improve efficiency, gain bigger market control, increase financial performance, improve profitability, and finally achieving overall firm success (Han \& Hwang, 2014; Wong et al., 2015 ).

Tourism and hospitality research has extended different concepts and models with rapid development on the topic of emotion and destination loyalty (Hosany et al., 2015). Increasing amount of studies have been undertaken to examine the intense reactions to events, place, restaurants and other parts of tourism and hospitality settings on the industry that is selling something much more profound than benefits of holidays as well as the way they make people feel (Ali et al., 2016; Han \& Jeong, 2013; Wong et al., 2015).

The dominant role of emotional experience in bringing advantage in term of destination loyalty such as increased satisfaction, improved retention, favourable word of mouth, increased relationships between tourist and host country has been repeatedly emphasized in recent studies (Wong et al.,2015; Ali et al.,2016; Baksi,2015; Han \& Jeong, 2013). Subsequently, more attentions have therefore been devoted to emotional experience subject and their roles in decision/loyalty formation especially in term of revisit intention to place and destination ( Baksi, 2015; Burgner et al., 2014; Su \& Hsu, 2013).

Even though substantial amount studies have been initiated in interpreting emotional experience among tourist, most of the past studies have applied concept and measures from psychology literature to explain how emotion works underpinning various theories such as Lazarus's Cognitive Appraisal Theory, Cannon Bard Theory and Schachter Singer Theory. While it is generally good to experience positive emotion in a place where one visit for a holiday, it does not appear to affect how given emotion can influence subsequent behavior of tourist which can indirectly benefit the destination managers. This denotes question about inadequacy of the past studies to incorporate characteristic of marketing in interpreting emotional experience to create value proposition and delivering tourism product in optimized values. In tourism marketing, tourist emotion should be examined to assess the role in decision making with the use of behavioral belief or attitude related theories to predict tourist subsequent intention to engage in a behavior at specific time and place.

In recent tourism research, emotions are often referred as affective responses elicited by consumption experiences (Ali, Amin \& Ryu, 2016). This concept was coined in agreement with the descriptions in early marketing studies propounding that consumption emotions are sets of immediate emotional responses induced during product/service usage (Richins 1997; Westbrook \& Oliver, 1991; Holbrook \& Hirschman, 1982; Havlena \& Holbrook, 1986). Self-report emotion measures have been also widely applied to understand tourist experiences (e.g. Plutchik, 1980; Izard, 1977; Mehrabian \& Russell, 1974; Watson, Clark \& Tellegen, 1988). Despite these strong foundations, in recent years, the applicability of psychological emotional scales in tourism studies fail to capture the richness of tourist and destination characteristics. For example, the emotion scales developed in psychology are context specific and does not necessarily suits the applicability in another setting.

Since Cohen's (1972) seminal work on tourist typology of organized mass tourist, the individual mass tourist, the explorer, and the drifter, a research stream has constructed tourist typologies using segmentation, classification, profiling, or clustering methods. Studies have empirically identified distinguishable patterns of tourist motivational responses, evaluation about experience and examine the relationships between tourists' emotional profiles and their destination loyalty.

Loureiro (2014) determined seven factors that characterized evaluation among tourist experiencing rural holidays in the South of Portugal: Hedonism (pleasurable feelings that excite oneself, fun, exciting, pleasant, and interesting sensations), involvement (to visit a desired place, to enjoy the activities), local culture (good impressions, friendly people and closely experience the ways of the local people), refreshment (liberating feeling, sense of freedom, refreshing, revitalized), meaningfulness (to do something meaningful and important, to learn about 
him/herself), knowledge (information, facts, or experiences known by an individual, exploration and educational) and novelty (cultural difference, newness, new culture, and exotic places). The study suggested positive emotions will generate positive memories which in turn play a key role in determining their intention to revisit.

Nawijn \& Fricke (2015) investigated the relationship between emotional experience and potential consequences in term of revisit intention among visitors to concentration camp memorial Neuengamme, Germany. This study showed emotional experience explicitly predicted repeat visit intention to concentration camp memorials as well as positive word of mouth. This consensus seems to extend the work of earlier researchers by providing empirical support for suggestions that emotional experience shape intended future travel behavior.

More countries had emphasized emotional experience in their offerings and tourism slogan such as Portugal, Germany, Taiwan, Malaysia, and Thailand. For example, Portugal positions the destination as offering a variety of experiences, including romance with "feel the passions" slogan throughout the country. Taiwan successfully uses the slogan "touch your heart" to convey a sense of warmth among potential tourists. Other countries highlight the 'surprising' component of the tourist experience. Campaigns such as Germany's "Simply Inspiring"; Netherland's "Surprising Cities"; Malaysia's "Truly Asia"; Thailand's "Amazing Thailand", Australia's "Experience Why...There is nothing like Australia" and Edinburgh's "Inspiring Capital" are good examples of emphasizing emotional experience in tourism offerings.

\section{CONCEPTUALIZATION OF EMOTION IN TOURISM RESEARCH}

Conceptualization of emotion is a contested issue in tourism research. Past literature offers two major approaches to classify emotion either by way of discrete (specific) or dimensional (valence based or combined) with respective underpinning theories (Havlena \& Holbrook, 1986; Li et al., 2015). In discrete approach, emotions are classified as fundamentally different construct while dimensional approach characterized in grouping. Ekman \& Cordaro, (2011) suggested human have innate set of basic emotions in discrete form which can be distinguished by facial expressions. The researchers contended that six basic emotions are anger, disgust, fear, happiness, sadness and surprise. Surprisingly the use in marketing studies are still limited due unavailability of discrete emotion via self-reported questionnaires. This approach is similar to previous emotion theories that categorize emotions as distinctive affective states and specific (Izard, 1977; Plutchik, 1980).
Dimensional approach has been widely used in tourism research borrowed from idea of consumption psychology. The dimensional approach gives a more parsimonious account of emotion as there is no need to distinguish between specific positive (e.g. joy, happiness and excitement) and negative (e.g. fear, regret and disappointment) emotions (Lazarus, 1991). Four commonly used psychological theories to assess emotions are Mehrabian and Russell's (1974) Pleasures, Arousal and Dominance (PAD) emotional state model, Izard's (1977) Differential Emotion Theory, Plutchik's (1980) Psychoevolutionary Theory, and Positive Affect and Negative Affect Schedule (PANAS) by Watson et al., (1988). Emotion theorist suggested sets of simple ways to categorize emotion based on a different theoretical framework which $\mathrm{h}$ as been widely employed by researchers in marketing and consumer behavior apart from psychology (Bagozzi et al., 1999; Westbrook \& Oliver, 1991).

Watson, Clark, \& Tellegen, (1988) after realizing the need for a reliable and comprehensive approach to capture emotions proposed the Positive and Negative Affect Schedule (PANAS) of positive affect (e.g., excited, inspired) and negative affect (e.g., upset, afraid). Positive and negative affect is two mood scale comprised with 10 descriptors each for positive mood and negative mood. PANAS is developed to deal with largely independent constructs of positive and negative affect.

Mehrabian and Russell (1974) proposed that human responses to environments are explained in terms of three independent bipolar dimensions: Pleasure (P); Arousal (A); and Dominance (D). The PAD emotional state model is a popular measure among researchers to determine the impact of hedonic nature related emotional experiences. In past tourism studies, PAD is the most commonly used framework to demonstrate emotional experience arising from the environment (Ali, Amin \& Ryu, 2016; Chang, Shu \& King, 2014).

Mehrabian and Russell (1974) focus on emotional response to environmental stimuli using three dimensions of pleasure-displeasure, arousalnon-arousal and dominance-submissiveness to measure feelings, moods and other related concepts. Their contention was based on environmental stimuli (S) will lead to emotional reaction $(\mathrm{O})$ which in turn drives the customer response (R) proposing as stimulus-organism- response $(\mathrm{S}-\mathrm{O}-\mathrm{R})$ framework. For example, respondents are often asked to indicate to what extent they feel pleasant or aroused. Sometimes 'pleasure' is measured by positive adjectives such as happy, pleased and satisfied, whereas individuals' level of arousal is indicated by items such as relaxed, calm and excited ( $\mathrm{Li}$ et al., 2015).

Izard's (1977) Differential Emotion Theory represent ten fundamental of human emotions consist 
of interest, joy, anger, disgust, contempt, sadness, fear, shame, guilt and surprise. Izard (1977)'s Differential Emotion Theory was also widely used by tourism researchers to conceptualize emotional experiences. For example, Han \& Jeong, (2013) incorporated four positive emotions and five negative emotions from Differential Emotion Theory and found that emotional experience mediated between perceived quality and behavioral intention in a restaurant setting. However, in many circumstances, this approach fails to find correspondences between discrete emotions and brain activity resulting in issues of generalizability.

Plutchik (1980) psycho-evolutionary theory of emotion is one of the most influential classification approaches for a general emotional experience. The theory proposed evolutionary approach to identify eight primary emotions consists of fear, anger, joy, sadness, acceptance, disgust, expectancy (anticipation) and surprise. The author posits that it is possible to mix descriptor pairs from basic emotions to assess the affective response of an experience. For example, emotion of delight consists of a combination of joy and surprise; love is a combination of joy and acceptance. It was based on Emotions Profile Index which contains 62 emotion descriptor pairs and responses are transformed into eight emotions.

\section{DISCUSSION}

Although it seems that research regarding emotional experience is well focused, the adaptation from psychology-based approach is criticized on validity ground and its employability in tourism research was debatable. Mehrabian and Russell's (1974) PAD is believed to be particularly adequate for assessing affective reactions to physical environments and its capacity to capture affective experiences related to product/service consumption is doubtful ( Chang et al., 2014). Many researchers omit the dominance dimension in PAD when studying consumer emotions based on expectations of low predictive value (Ali et al., 2016). Whereas for Izard's (1977) Differential Emotion Theory and Plutchik's (1980) psychoevolutionary theory of emotion was criticized on the predominance of negative emotion with inconsistent and debatable descriptors (Prayag et al.,2017).

In order to overcome the shortcomings of the previous approach to comprehend emotions in consumer behavior, Richins (1997) developed the Consumption Emotion Set (CES) employing 47 emotion descriptors grouped into 16 dimensions to study on emotion encountered during consumption experience. The dimensions consist of anger, worry, sadness, fear, shame, romantic love, excitement, optimism, joy, surprise, discontent, love, envy, loneliness, peacefulness and contentment. However,
CES was criticized on the question of discriminant validity in tourism studies (Knobloch et al., 2016) .

A study by Han et al., (2010) on measuring emotion in restaurant settings adapting the CES also failed on discriminant validity. This in turn questions on the generalizability of Richin's (1997) Consumption Emotion Set in other settings. Furthermore, it also indicates limitation these theories suffers from generalizability when applied to different settings of emotional experience. This is due to the nature of consumer emotional experiences which is very broad and context specific. This coincides with the example by Richin (1997) that "emotions that arise in the context of intimate interpersonal relationships are likely to differ in intensity and quality from emotions experienced when buying a pair of shoes". Consequently, mixed evidence and the lack of an appropriate measure to capture consumption related emotional experience, have hindered theoretical progress in marketing research.

As far as tourism is concerned, there are attempts to identify the structure of emotional experience elicited during tourism-related events and responses toward destinations but there is still a question of reliability (Ma et al., 2016; Burgner et al., 2014). This is due to the generalizability resulting from adopting the concept of emotion directly from common psychological approach as discussed above.

This was congruent as argued by Prayag et al., (2017) that most past analyses relied primarily on exploratory analytic procedures such as exploratory factor analysis to uncover the underlying structure of basic emotions. Even though these techniques are useful for discovering conceptual congruence among subsets of basic emotions, they reveal shortcomings of subjective interpretations of the dimensions and an inability to replicate across study contexts.

This issue has been inherent in most tourism studies that have adopted the aforementioned approach. This is clearly evidenced and manifested when measures relating to affective states in tourism and hospitality often include irrelevant descriptors contrary with tourists' emotional experiences as argued by Kumar \& Nayak, 2015). This is because emotions of the same valence have different effects on judgment, decision-making, satisfaction and loyalty (Nawijn \& Fricke, 2015; Barnes et al., 2016) depending on the context, sequence of events and intensity of the feelings.

\section{CONCLUSION}

In recent years, researchers in tourism and hospitality studies have concerned seriously on the conceptualization of emotional experience in the process to develop strong long term engagement leveraging from affective element to predict loyalty towards destination among tourist. However the available studies are still clouded by mixed evidence that does not clearly distinguish appropriate approach 
to quantify the intensity of arousal that can conclusively predict loyalty. Among the reason is the unsuitable use of psychology approach to describe emotion elicited during tourism experience that fails to take into account appropriate response to events and behavioral impact.

The direction of this conceptual paper apart from extending the existing body of knowledge, could provide logical argument for future studies in tourism marketing to employ context specific emotional experience adopting dimensional approach based on respective research settings to accurately account the strength of emotional experience to predict loyalty to destination. The future studies could further draw a model integrating emotions, experience and expression comprehensively taking into the appraisal element with pertinent marketing theoretical approach. Likewise, the accurate types of emotional experience provide indication to managers to create and manage the site experience appropriately. The proposed design although yet to empirically tested has potential academic and practical implication as more destination marketers are looking for appropriate approach to incorporate emotional content in their offerings.

\section{REFERENCES}

1. Ali, F., Amin, M., \& Ryu, K. (2016). The role of physical environment, price perceptions, and consumption emotions in developing customer satisfaction in Chinese resort hotels. Journal of Quality Assurance in Hospitality \& Tourism, 17(1), 45-70.

2. Argan, M. T., Argan, M., \& Akyildiz, M. (2014). Dimensions of Consumption Emotions: Turkish Consumers' Experiences. Journal of Marketing and Management, 5(1), 136.

3. Awang, K. W., Hassan, M., \& Abdullah, A. R. (2015). Tourism Landscape: Understanding the Swiftlet "Hotel" Phenomenon. Sains Humanika, 5(2).

4. Baksi, A. K. (2015). Craftourism: moderating emotional-bonding with destination and its loyalty. Asia-Pacific Journal of Innovation in Hospitality and Tourism APJIHT, 159.

5. Barnes, S. J., Mattsson, J., \& Sorensen, F. (2016). Remembered experiences and revisit intentions: $A$ longitudinal study of safari park visitors.Tourism Management, 57, 286-294.

6. Burgner, K. D., Ravulaparthy, S., \& Goulias, K. (2014). Place happiness: Its constituents and the influence of emotions and subjective importance on activity type and destination choice. In Transportation research board 93rd annual meeting (No. 14-0074).

7. Caru, A., \& Cova, B. (2014). The Design of Consumer Experiences: Managerial Approaches for Service Companies. In Managing Consumer Services (pp. 91-108). Springer International Publishing.

8. Chang, C. H., Shu, S., \& King, B. (2014). Novelty in theme park physical surroundings: An application of the stimulus-organism-response paradigm. Asia Pacific Journal of Tourism Research, 19(6), 680-699.

9. Cohen, J. B., \& Areni, C. S. (1991). Affect and consumer behavior.

10. Cohen, E. (1972). Toward a sociology of international tourism. Social research, 164-182.

11. Donnelly, G., Ksendzova, M., \& Howell, R. T. (2013). Sadness, identity, and plastic in overshopping: The interplay of materialism, poor credit management, and emotional buying motives in predicting compulsive buying.Journal of Economic Psychology, 39, 113-125.

12. Ekman, P., \& Cordaro, D. (2011). What is meant by calling emotions basic. Emotion Review, 3(4), 364-370.

13. Faullant, R., Matzler, K., \& Mooradian, T. A. (2011). Personality, basic emotions, and satisfaction: Primary emotions in the mountaineering experience. Tourism Management, 32(6), 1423-1430.

14. Grappi, S., \& Montanari, F. (2011). The role of social identification and hedonism in affecting tourist re-patronizing behaviours: The case of an Italian festival. Tourism Management, 32(5), 1128-1140.

15. Gyung Kim, M., \& Mattila, A. S. (2013). Does a surprise strategy need words? The effect of explanations for a surprise strategy on customer delight and expectations. Journal of Services Marketing, 27(5), 361-370.

16. Han, H., \& Hwang, J. (2014). Investigation of the volitional, non-volitional, emotional, motivational and automatic processes in determining golfers' intention: Impact of screen golf. International Journal of Contemporary Hospitality Management, 26(7), 1118-1135.

17. Han, H., \& Jeong, C. (2013). Multi-dimensions of patrons' emotional experiences in upscale restaurants and their role in loyalty formation: Emotion scale improvement. International Journal of Hospitality Management, 32, 59-70.

18. Han, H., Back, K. J., \& Barrett, B. (2010). A consumption emotion measurement development: a full-service restaurant setting. The Service Industries Journal, 30(2), 299-320.

19. Havlena, W. J., \& Holbrook, M. B. (1986). The varieties of consumption experience: comparing two typologies of emotion in consumer behavior. Journal of consumer research, 13(3), 394-404.

20. Hosany, S., \& Gilbert, D. (2010). Measuring tourists' emotional experiences toward hedonic holiday destinations. Journal of Travel Research, 49(4), 513-526.

21. Izard, C.E. (1977). Human Emotions. Plenum, New York.

22. Kerr, M. M., \& Price, R. H. (2018). "I Know the Plane Crashed": Children's Perspectives in Dark Tourism. In The Palgrave Handbook of Dark Tourism Studies (pp. 553-583). Palgrave Macmillan, London.

23. Knobloch, U., Robertson, K., \& Aitken, R. (2016). Experience, Emotion, and Eudaimonia A Consideration of Tourist Experiences and Well- 
being. Journal of Travel Research, 0047287516650937.

24. Kumar, V., \& Nayak, J. K. (2015). Examining the relationship between patterns of emotional responses, satisfaction, and loyalty. Anatolia, 114.

25. Lazarus, R. S. (1991). Progress on a cognitivemotivational-relational theory of emotion. American psychologist, 46(8), 819.

26. Li, S., Scott, N., \& Walters, G. (2015). Current and potential methods for measuring emotion in tourism experiences: A review. Current Issues in Tourism, 18(9), 805-827.

27. Loureiro, S. M. C. (2014). The role of the rural tourism experience economy in place attachment and behavioral intentions. International Journal of Hospitality Management, 40, 1-9.

28. Ma, J., Campos, A. C., Li, S., Gardiner, S., \& Scott, N. (2016). Attention, emotion and hedonic service experiences: Managing and delivering services in the Asian Century. Worldwide Hospitality and Tourism Themes, 8(1), 53-60.

29. Mehrabian, A., \& Russell, J. A. (1974). An approach to environmental psychology. the MIT Press.

30. Nawijn, J., \& Fricke, M. C. (2015). Visitor emotions and behavioral intentions: The case of concentration camp memorial Neuengamme.International Journal of Tourism Research, 17(3), 221-228.

31. Oliver, R. L. (1999). Whence consumer loyalty?. the Journal of Marketing, 33-44.

32. Othman, P., \& Rosli, M. M. (2011). The impact of tourism on small business performance: Empirical evidence from Malaysian islands. International Journal of Business and Social Science, 2(1).

33. Plutchik, R. (1980). Emotion: A psychoevolutionary synthesis. Harpercollins College Division

\section{Authors Profile}

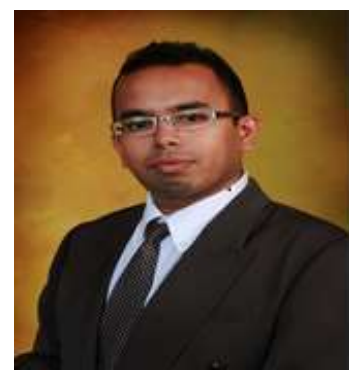

Abdul Hazif Abdul Hamid began his career in Malaysian Administrative and Diplomatic Service, a premier civil service of Malaysia and served various positions in economic planning and development for nearly 20 years. His current research focus is experiential marketing, place management, destination marketing, tourism and hospitality management. $\mathrm{He}$ is currently engaged as graduate researcher with Faculty of Entrepreneurship and Business, Universiti Malaysia Kelantan, Malaysia.

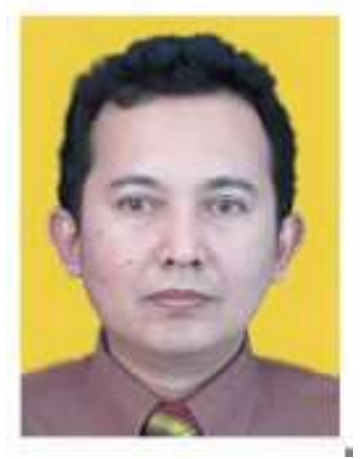

Mohd Rosli Bin Mohamad is the Director of Development and Senior Professor of Economics at Faculty of Entrepreneurship and Business, Universiti Malaysia Kelantan (UMK). He has more than 25 years of teaching, research, and advisory experience. His research focuses on the fields of performance management in Small Medium Enterprises, tourism management and innovation. He is also editor-in chief of Journal of Entrepreneurship and Business, a double-blind, peer-reviewed and open-access journal. 